Психология. Журнал Высшей школы экономики.

2018. T. 15. № 1. C. 10-21. DOI: 10.17323/1813-8918-2018-1-10-21

\title{
ИНТЕЛЛЕКТ И УСПЕШНОСТЬ СТРАТЕГИЙ ПРОГНОЗИРОВАНИЯ ПРИ ВЫПОЛНЕНИИ АЙОВА-ТЕСТА (IGT)
}

\author{
Т.В. КОРНИЛОВА ${ }^{\mathrm{a}}$ М.А. ЧУМАКОВА ${ }^{\mathrm{b}}$, С.А. КОРНИЛОВ
}

\begin{abstract}
${ }^{a}$ МГУ имени М.В. Ломоносова, 119991, Россия, Москва, Ленинские горы, д. 1
${ }^{\circ}$ Национальный исследовательский университет «Высиая икола әкономики», 101000, Москва, ул. Мяспицкая, Ә. 20

${ }^{\circ}$ Санкт-Петербургский государственный университет, 199034, Санкт-Петербург, наб. Макаровa, ว. 6
\end{abstract}

\begin{abstract}
Резюме
Айова-тест (IGT) используется в качестве удобной модели изучения процессов прогнозирования. IGT применялся преимущественно для получения данных в пользу гипотезы «соматических маркеров» А. Дамасио. В зарубежных исследованиях выделялась ведуцая роль эмоциональных компонентов регуляции по сравнению с интеллектуальными. В нашем исследовании, выполненном на выборке взрослых испытуемых ( $\mathrm{n}=116$ ) из группы нормы, измерялся вербальный, флюидный и общий интеллект. На основе регрессионного анализа показано, что предиктором успешности выборов в IGT выступает общий интеллект, причем в трех последних блоках игры. Вербальный интеллект также стал положительным предиктором предпочтений «хороших» колод (в блоке 4). Однако интеллект не является предиктором на самом первом этапе игровой ситуации, когда она максимально неопределенная и когнитивные ориентиры еще не выявлены. Полученные результаты отражают динамику компонентов принятия решения, связанных с изменением ориентировки в условиях IGT и изменением регуляции со стороны интеллекта. Более высокий интеллект позволяет испытуемым строить корректные репрезентации задачи, отдавать предпочтение «хорошим» колодам и выигрывать больше условных денег в заданной ситуации. Предполагаемые концепцией «соматических маркеров» влияния со стороны эмоциональной обратной связи могут превалировать именно в начале игровой ситуации, когда прогностическая активность еще не оформлена в когнитивные ориентиры. При становлении же игровых стратегий на большом числе проб неопределенность ситуации уменьшается и на первый план выходят когнитивные компоненты ориентировки в вероятностной среде.
\end{abstract}

Ключевые слова: интеллект, прогнозирование, игровая задача Айова, Айова-тест.

В исследованиях предвосхищений в ситуациях неопределенности традиционно дань первенства отда- валась интуиции и вероятностному прогнозированию. Последнее могло рассматриваться как в контекстах

Исследование поддержано грантом РГНФ, проект № 15-06-10404. 
работы так называемой когнитивной Системы 1 (Канеман, 2013), так и в более широком контексте рассмотрения интеллектуально-личностных различий в опережающей активности со стороны образа мира (Смирнов и др., 2016). В последние десятилетия было показано, что в ситуациях неопределенности при принятии решений большей эффективностью характеризуются стратегии, сочетающие рациональный анализ и интуицию (Степаносова, Корнилова, 2006). Соответствующие конструкты могут быть операционализированы через предпочитаемые стилевые характеристики принятия решений (Корнилова, Разваляева, 2017; Epstein et al., 1996). В целом же принятие решений стало той ведущей областью исследований, в которой утвердилось представление о необходимом взаимодействии когнитивной и эмоциональной, рациональной и интуитивной подготовки прогнозов и выборов в условиях неопределенности (Корнилова, 2016а; Чумакова, 2013; Hastie, Dawes, 2010).

Важной характеристикой при изучении прогнозирования является фактор задач, поскольку включение в построение прогнозов предметного содержания существенно для становления профилей регуляции предвосхищений и решений в условиях неопределенности. Изучение вклада когнитивных способностей в процессы прогнозирования в последние несколько десятилетий стало уходить на второй план, а собственно учет эмоциональной сферы стал чаще попадать в фокус внимания исследователей после того, как A. Дамасио выдвинул гипотезу «соматических маркеров» (Damasio et al., 1996). Маркеры при этом пони- маются как возникающие при определенных сигналах висцеральные реакции, построенные на основе предыдущего индивидуального опыта и субъективно переживаемые как «Подозрения», «внутри что-то екнуло» и пр. (Медведева и др., 2013). Популяризации этой концепции во многом способствовало то, что она служит теоретической поддержкой выявления механизмов нарушения обратной связи для разных клинических групп на основе единой экспериментальной модели при использовании набора заданий, получивших название игровой задачи Айова, или Айова-теста, - Iowa Gambling Task (IGT) (Bechara et al., 2005).

Концепция образа мира фокусирует иной, чем только обратная связь от чувственного индивидуального опыта, аспект прогнозирования: опережающую прогностическую активность субъекта, направляемую амодальными, а значит, не обязательно переживаемыми чувственно - глубинными структурами (включающими, в частности, индивидуальную представленность значений). Вместе с тем психология принятия решений выделяет и универсальные закономерности ориентировки человека в ситуации неопределенности и риска, предполагая в качестве диагностической проблемы анализ того, какие процессы выходят на ведущий уровень в динамических регулятивньх системах, обеспечивающих становление прогнозов, оценок и выборов человека (Корнилова, 2016а, б).

Соотношение прогнозирования и принятия решений - при прагматической цели достижения выигрыша - успешно исследовалось на экспериментальной модели Айова-теста, 
упомянутого выше. IGT требует от испытуемого выбирать карты из 4 колод, различающихся по вероятностной структуре выигрышей и проигрышей, которую испытуемый выявляет в процессе эксперимента (колоды предъявляются «рубашкой вверх», и испытуемый узнаёт на каждом ходу, сколько игровых денег он выиграл или проиграл). На неклинических выборках предикторы индивидуальных различий в успешности выполнения IGT исследовались редко (Buelow, Suhr, 2009). Хотя имеются работы о связях стратегий в IGT с эмоциональной сферой (что неудивительно ввиду популяризации методики в связи с разработкой идей соматических маркеров), связи c интеллектом изучались реже. В 2014 г. К. Вебб (Webb et al., 2014) отмечал, что известно только одно исследование с одновременным фиксированием свойств IQ и эмоционального интеллекта на неклинической выборке. Указанное исследование обнаружило, что IQ оказывается лучшим предиктором успешности выполнения Айова-теста, а значит, выполнение IGT задействует в большей степени когнитивные факторы, чем эмоциональные (по крайней мере, больше, чем эмоциональный интеллект). В исследовании Х. Демари (Demaree et al., 2010) для оценки когнитивных способностей на студенческой выборке применялась вербальная шкала Милла-Хилла, а не традиционные «золотые стандарты» тестов интеллекта (шкалы Векслера и Стэнфорда-Бине). В исследовании же К. Вебба применялся тест Векслера для взрослых (WASI) на материале выборки испытуемых, которые различались как по возрас- ту, так и по образованию. Авторы не нашли связи выполнения IGT с эмоциональным интеллектом, но установили значимые корреляции с общей шкалой IQ, с вербальным и практическим интеллектом (Webb et al., 2014).

Мы разделяем предположение, что интеллект должен предсказывать эффективность выполнения заданий IGT, поддерживая тем самым гипотезу о выполнении IGT на основе развертывания преимущественно когнитивной ориентировки. Тем не менее мы хотели бы отметить, что именно процессы предвосхищения и прогнозирования наименее представлены в тех видах активности, которые требуются для выполнения тестов на интеллект. Это противоречие решается, если рассматривать IGT как задание на обучение, в котором испытуемый встречается с необходимостью устанавливать и постоянно обновлять вероятностную репрезентацию структуры выигрышей и проигрышей в задаче. Ранее мы показали связи между стратегиями в IGT и личностными свойствами толерантности-интолерантности к неопределенности (Kornilov et al., 2015), психологическая разумность (Kornilova, Razvalyaeva, 2016) и др. Таким образом, можно говорить о системной множественной регуляции выполнения IGT и со стороны личностных, и со стороны когнитивных процессов. Их взаимодействие и представлено в разных типах стратегий.

Осторожные и рациональные стратегии можно противопоставлять в IGT рискованным или хаотичным по параметрам соотношения полученных выигрышей и проигрышей с частотой и последовательностью смены колод. 
Реализация испытуемым рискованной или хаотичной стратегии не обязательно должна рассматриваться как нарушение ориентировки на собственные эмоциональные состояния, как предполагает гипотеза А. Дамасио. За выбором рискованных колод могут стоять и исследовательская активность, проявляющаяся в стремлении испытуемого понять закономерности, лежащие в основе задачи, и желание максимизировать выигрыш на последних пробах.

Целью нашего исследования стало выявление связи между интеллектом (вербальным/кристаллизованным и флюидным) и показателями успешности выполнения IGT, а также вклада разных видов ориентировки в становление стратегий испытуемых на разных этапах игры.

Исследование выполнялось на выборке взрослых из группы «нормы» с разной профессиональной принадлежностью.

\section{Метод}

Уиастиики исследования. В исследовании приняли участие 116 взрослых испытуемых (56 женщин и 60 мужчин). Средние по возрасту: для женщин $\mathrm{M}=31.07$, для мужчин $\mathrm{M}=$ 33.82; группы не различались по возрасту статистически $(p>0.05)$.

Игровая задача Айова (или Айова-тест) применялась в адаптированном С.А. Корниловым русскоязычном варианте компьютеризированного стандарта условий IGT, разработанного Р. Грасманом и Е. Вaгенмейкерсом (Grasman, Wagenmakers, 2005). Последовательность из 100 проб при анализе разбивалась на 5 блоков по 20 проб, чтобы про- следить изменение связей на разных этапах - при большей и меньшей неопределенности ситуации.

Задача испытуемого - получить своими выборами как можно больше условных «денег» в конце игры. При каждом выборе карта может привести к получению или потере условной денежной суммы. Колоды неодинаковы с точки зрения баланса выигрышей и проигрышей. В двух колодах представлены карточки высокого риска - они дают относительно высокие выигрыши (\$100), но и разорительные штрафы (до -\$1250), и поэтому их предпочтение в долговременной перспективе ведет к проигрышу; две другие колоды дают возможность постепенно накапливать небольшие суммы (\$50) и небольшие штрафы, но в долговременной перспективе ведут к выигрышу. Предполагается, что игроки постепенно научаются ориентироваться в вероятностной структуре ситуации неопределенности: они понимают различие частот выпадения сумм разной величины при выборе той или иной колоды.

Зависимые переменные IGT: общий выигрыш, пропорция-предпочтение «хороших» колод в каждом блоке и общее предпочтение «хороших» колод.

Диагностика интеллекта. Для оценки флюидного интеллекта использовались субтесты Решение матриц и Трехмерное вращение из тестовой батареи ICAR (Condon, Revelle, 2014). Сырые баллы по субтестам были стандартизованы, усреднены и трансформированы в IQ-шкалу.

Вербальный интеллект оценивался по двум вербальным шкалам из тестовой батареи ROADS (Корнилов, Григоренко, 2010). 
Общий интеллект оценивался по всем четырем использованным субтестам.

\section{Результаты}

Для анализа данных использовался линейный регрессионный анализ, в котором в качестве зависимых переменных выступали показатели прибыли и предпочтения «хороших» колод в каждом блоке и кумулятивно за всю игру. Тестировались две модели: Модель 1 включала в качестве предикторов пол, возраст, взаимодействие между ними, флюидный и вербальный IQ; Модель 2 включала те же демографические предикторы и общий IQ.

Для прибыли в каждом блоке обе модели продемонстрировали значимый вклад интеллекта: Модель 1 выявила значимый вклад в прибыль флюидного интеллекта в блоке 2 $\left(F(\mathrm{df})=1.896(104), p=0.048, \Delta^{1} \mathrm{Ad}-\right.$ justed $\left.R^{2}=0.03, \beta=10.56, p=0.024\right)$; те же результаты были получены для Модели $2(\mathrm{~F}(\mathrm{df})=1.929(105), p=0.050$, $\triangle$ Adjusted $\left.R^{2}=0.02, \beta=11.00, p=0.041\right)$.

Для общего выигрыша было установлено, что в Модели 1 значимым предиктором является вербальный $\mathrm{IQ}(\mathrm{F}(\mathrm{df})=3.557$ (104), $p<0.001$, $\triangle$ Adjusted $R^{2}=0.10, \beta=23.78, p=$ $=0.005)$. Модель 2 также показала значимое влияние общего IQ $(F(\mathrm{df})=$ $=3.723$ (105), $\mathrm{p}<0.001, \triangle$ Adjusted $\left.R^{2}=0.09, \beta=23.45, \mathrm{p}<0.001\right)(\mathrm{cm}$. рисунок 1). Как видно из рисунка 1 , большей части испытуемых с высоким общим IQ в течение всей игры, за исключением первых 20 ходов, удавалось накапливать прибыль, в то время как существенная часть испытуемых с низким и средним уровнем IQ систематически оставалась в проигрыше 2.

Puсyиок 1

Накопленный средний выигрыш (по блокам) у групп с разным уровнем интеллекта

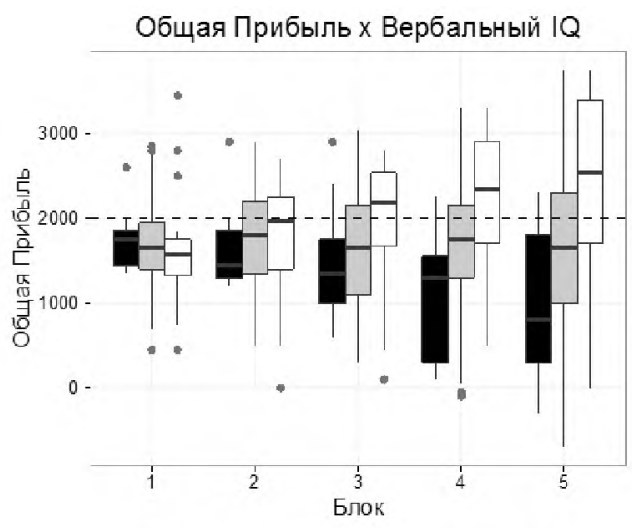

Вербальный IQ 85 官85-115 它>115

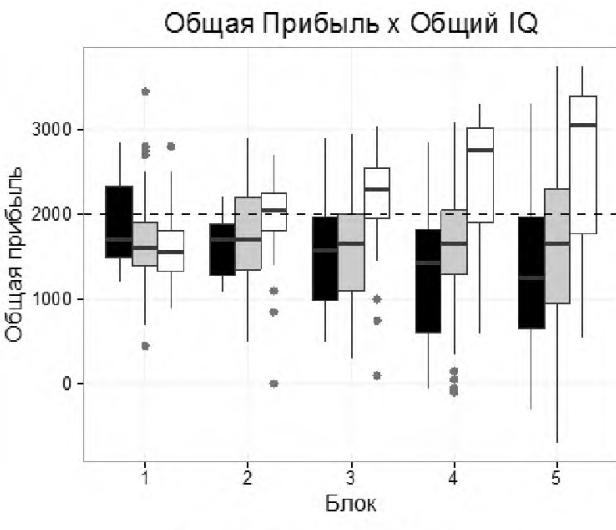

Общий IQ <85 Њ85-115 ウ>115

${ }^{1}$ Здесь и далее изменение Adjusted $R^{2}$ при добавлении показателей IQ к демографическим предикторам.

${ }^{2} 2000$ - сумма перед началом игры. 
Вербальный IQ оказался положительным предиктором предпочтений «хороших» колод в блоке $4(F(\mathrm{df})=$ $=2.014(104), p=0.034, \triangle$ Adjusted $R^{2}=$ $=0.06, \beta=0.23, p=0.023)$. Общий IQ оказался положительным предиктором предпочтения колод в трех из пяти блоков (блок $3: F(\mathrm{df})=2.985$ (105), $p=0.002, \triangle$ Adjusted $R^{2}=0.05$, $\beta=0.18, p=0.008 ; 4: F(\mathrm{df})=2.092$ (105), $p=0.031, \triangle$ Adjusted $R^{2}=0.06$, $\beta=0.22, p=0.007 ;$ блок $5: F(\mathrm{df})=$ $=2.247$ (105), $p=0.020, \Delta$ Adjusted $\left.R^{2}=0.04, \beta=0.18, p=0.017\right)$.

Мы не обнаружили значимого вклада вербального IQ в накопленное предпочтение «хороших» колод в течение эксперимента, как и флюидного. Однако мы установили, что общий IQ является значимым предиктором для этого показателя в блоках с 3 по 5 (блок $3: F(\mathrm{df})=2.001$ (105), $p=0.040, \triangle$ Adjusted $R^{2}=0.03$, $\beta=0.30, p=0.030$; блок 4: $F(\mathrm{df})=$ $=2.272(105), p=0.019, \triangle$ Adjusted $R^{2}=$ $=0.05, \beta=0.52, p=0.010$; блок 5 :
$F(\mathrm{df})=2.610(105), p=0.007, \triangle \mathrm{Ad}-$ justed $R^{2}=0.04, \beta=0.70, p=0.007$ ).

Рисунок 2 демонстрирует значимые эффекты, полученные в регрессионном анализе. Начиная со второго блока, у испытуемых с высоким уровнем общего IQ систематически увеличивается количество выборов «хороших» колод, обеспечивающих выигрыш в долгосрочной перспективе. При этом испытуемыми с общим IQ ниже 115 баллов на протяжении всей игры предпочтение отдается «плохим» колодам, дающим больший сиюминутный выигрыш, но приводящим к проигрышу в финале.

\section{Обсуждение результатов}

Полученные в нашем исследовании результаты отражают динамику компонентов принятия решения, связанных с изменением ориентировки в условиях IGT и изменением регуляции со стороны интеллекта. Более высокий интеллект позволяет

Рисунок 2

Предпочтение «хороших» колод у групп с разным уровнем интеллекта

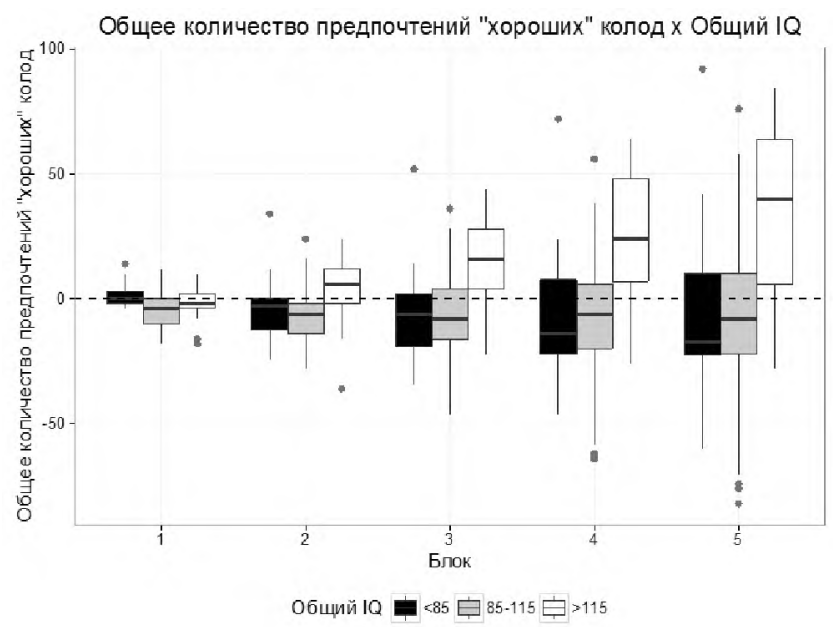


испытуемым строить корректные репрезентации задачи, отдавать предпочтение правильным колодам и выигрывать больше условных денег в заданной ситуации, требующей прогностической активности от участника.

Наше исследование показало, что общий интеллект является ключевым фактором успешности принятия решений, требуюших выявления скрытых закономерностей. Мы не обнаружили ощутимого вклада флюидного интеллекта в успешность, хотя предполагали это. Также результаты свидетельствуют о том, что общий интеллект оказался лучшим предиктором успешности по сравнению с изолированным вербальным интеллектом. Пока мы не сталкивались с обсуждением того, в какой степени вербальные и невербальные компоненты включены в становление образа ситуации в IGT. Это позволяет говорить о необходимости проверки вклада общих vs специфических когнитивных способностей в регуляцию принятия решений и прогнозирования.

Отсутствие вклада любого вида интеллекта в первой двадцатке проб, т.е. на этапе максимальной неопределенности ситуации, позволяет предполагать, что либо в построении образа задачи (образа ситуации) включены и неинтеллектуальные факторы, либо 20 проб (только первого блока) недостаточно для надежной оценки начальных процессов ориентировки.

Во введении было сказано, что нам удалось продемонстрировать значимый вклад толерантности-интолерантности к неопределенности в показателе обучения при лонгитюд- ном анализе данных IGT (Kornilov et al., 2015). Толерантность регулировала начальный уровень принятия риска в условиях неопределенности, а интолерантность была связана с более низкой исследовательской активностью после проигрышей (в частности, с более редкой сменой колод). Ранее нами было показано, что с уровнем интеллекта толерантность к неопределенности не связана, а интолерантность связана отрицательно (Корнилова, 2016а). Таким образом, мы принимаем предположение о влиянии динамических изменений в отношении к неопределенности на разных этапах стратегий в IGT, но должны - согласно нашим результатам - предполагать ключевую роль влияния когнитивных процессов (как отличающих лиц с более высоким уровнем интеллекта) в игровых стратегиях (при снижении неопределенности от второго к пятому блоку).

В отдельной работе нами были показаны связи выраженности свойств Темной триады (нарциссизма, макиавеллизма, психопатии) со стратегиями в IGT (Красавцева, Корнилова, 2016). В частности, именно в первом блоке выборов выраженность макиавеллизма сопутствовала предпочтению выигрышных колод (но не суммы выигрыша), а повышение нарциссизма снижало предпочтения прибыльной, но рискованной колоды. Это поддерживает наше предположение о вкладе в регуляцию стратегий при максимальной неопределенности преимушественно не со стороны когнитивных ориентиров (которые на первом этапе еще не выявлены), а со стороны личностных свойств, включающих общность нестабильного эмоционального ядра, 
связываемого с Темной триадой (Paulhus, Williams, 2002).

Таким образом, наши исследования уточняют, что предполагаемые концепцией «соматических маркеров» влияния со стороны эмоциональной обратной связи могут превалировать именно в начале игровой ситуации, когда прогностическая активность еще не оформлена в когнитивные ориентиры. При становлении же игровых стратегий на большом числе проб неопределенность ситуации уменьшается и на первый план выходят когнитивные компоненты ориентировки в вероятностной среде.

\section{Выводы}

Полученные данные о включенности факторов интеллекта в регуля- цию стратегий в Айова-тесте ставят под сомнение превалирование процессов эмоциональной регуляции (постулируемой в гипотезе «соматических маркеров).

Общий интеллект выступает предиктором успешности стратегий в Айова-тесте, что не решает, однако, проблему двузначности интерпретации его вклада в регуляцию выборов со стороны обучения или когнитивной способности.

Опираясь на отсутствие вклада интеллекта в успешность IGT в блоке 1 и продемонстрированный другими нашими исследованиями вклад в успешность в этом блоке ряда личностных свойств, можно сделать предположение, что решения при наиболее высоком уровне неопределенности регулируются в большей степени личностными особенностями.

\section{Литература}

Канеман, Д. (2013). Думай медленно... решай быстро. М.: АСТ.

Корнилов, С. А., Гриторенко, Е. Л. (2010). Методический комплекс для диагностики академических, творческих и практических способностей. Психологичекий журнал, 31(2), 90-103.

Корнилова, Т. В. (2016а). Интеллектуально-лииностный потенииал человека в условиях неопределениости и риска. СПб.: Нестор-История.

Корнилова, Т. В. (2016б). Психология выбора как мыслительное и личностное опосредствование преодоления неопределенности. Психологииеский журнал, 37(3), 113-124.

Корнилова, Т. В., Разваляева, А. Ю. (2017). Апробация русскоязычного варианта полного опросника С. Эпстайна «Рациональный-Опытный» (Rational-Experiental Inventory). Психологический журнал, 38(3), 92-107. doi:10.7868/S0205959217030084

Красавцева, Ю. В., Корнилова, Т. В. (2016). Свойства Темной триады в регуляции стратегий принятия решений (на материале игровой задачи Айова - IGT). Вестник Московского государственного областного упиверситета. Серия: Психологические науки, 2, 2-33. doi:10.18384/2310-7235-2016-2-22-33

Медведева, Т. И., Ениколопова, Е. В., Ениколопов, С. Н. (2013). Гипотеза соматических маркеров Дамасио и игровая задача (IGT): обзор. Психологиеские исследования, 6(32), 10. Режим доступа: http://psystudy.ru/index.php/num/2013v6n32/912-medvedeva32.html

Смирнов, С. Д., Чумакова, М. А., Корнилова, Т. В. (2016). Образ мира в динамическом контроле неопределенности. Вопросы психологии, 4, 3-13. 
Степаносова, О. В., Корнилова, Т. В. (2006). Мотивация и интуищия в регулящии вербальных прогнозов при принятии решений. Психологический журиал, 27(2), 60-68.

Чумакова, М. А. (2013). Личностная регуляция рационального выбора: развитие идеи единства интеллекта и аффекта. Психологиеский журнал, 34(3), 119-125.

Ссылки на зарубежные источники см. в разделе References после англоязыиного блока.

Корнилова Татьяна Васильевна - профессор, факультет психологии, Московский государственный университет имени М.В. Ломоносова, доктор психологических наук.

Сфера научных интересов: психология мышления и принятия решений, психология личности, психология риска, саморегуляция, интеллектуально-личностный потенциал.

Контакты: tvkornilova@mail.ru

Чумакова Мария Алексеевна - доцент, департамент психологии, факультет социальных наук, Национальный исследовательский университет «Высшая школа экономики», кандидат психологических наук.

Сфера научных интересов: принятие решений, интеллектуально-личностный потенциал, индивидуальные различия в когнитивных способностях и функциях.

Контакты: mchumakova@hse.ru

Корнилов Сергей Александрович - научный сотрудник, факультет психологии, СанктПетербургский государственный университет, кандидат психологических наук.

Сфера научных интересов: психометрика, интеллект, расстройства развития.

Контакты: sa.kornilov@gmail.com 


\title{
Intelligence and Successful Prognostic Strategies in Iowa Gambling Task (IGT)
}

\author{
T.V. Kornilova ${ }^{\mathrm{a}}$, M.A. Chumakova ${ }^{\mathrm{b}}$, S.A. Kornilov ${ }^{\mathrm{c}}$ \\ ${ }^{a}$ Lomonosov Moscow State University, GSP-1, Leninskie Gory, Moscow, 119991, Russian Federation \\ ${ }^{b}$ National Research University Higher School of Economics, 20 Myasnitskaya Str., Moscore, 101000, \\ Russian Federation \\ 'Saint-Petersburg State University, Saint-Petersburg, 6 Makarova embankment, 199034, Russian \\ Federation
}

\begin{abstract}
Iowa Gambling Task (IGT) is frequently used as a convenient model for studying processes of decision making and prognosis. Clinical studies generally provide support for Damasio's "somatic markers" hypothesis. Many studies stress out the leading role of emotional components of IGT performance regulation in comparison with intellectual components. In our study the verbal, fluid and general intelligence were measured on a sample of adult subjects $(n=116)$ from the non-clinical group. Using linear regression, we showed that intelligence was a significant predictor of the successful decision making in IGT, in particular in three last blocks. Verbal IQ also became a positive predictor of the preferences of "good" decks (in block 4). However, intelligence did not significantly predict success in the earliest stage of the game, when the game was the most undefined and cognitive markers haven't been revealed yet. Thus obtained results reflect the dynamics of decision-making components and changing in the intelligence impact in decisionmaking regulation. Higher intelligence provides more accurate cognitive representations of the task, choices of correct decks and as a result gaining more money in the task. We conclude by noting that the emotional influences and regulation predicted by the somatic marker hypothesis probably have the leading role at the earliest stages of decision making under uncertainty, where prognostic activity is not yet defined through cognitive markers. Uncertainty reduction related to the formation of game strategies through the large number of trials allows cognitive factors of adaptation and orienting in probabilistic environment take the leading role in decision-making regulation in IGT.
\end{abstract}

Keywords: intelligence, prognosis, Iowa Gambling Task, IGT.

\section{References}

Bechara, A., Damasio, H., Tranel, D., \& Damasio, A. R. (2005). The Iowa Gambling Task and the somatic marker hypothesis: some questions and answers. Trends in Cognitive Sciences, 9(4), 159-162. doi:10.1016/j.tics.2005.02.002.

Buelow, M. T., \& Suhr, J. A. (2009). Construct validity of the Iowa gambling task. Neuropsychology Reriere, 19(1), 102-114. 
Chumakova, M. A. (2013). Lichnostnaja reguljacija racional'nogo vybora: razvitie idei edinstva intellekta $i$ affekta [Personal regulation of rational choice: developing the idea of the unity of intellect and affect]. Psikhologicheskii Zhumal, 34(3), 119-125.

Condon, D. M., \& Revelle, W. (2014). The International Cognitive Ability Resource: Development and initial validation of a public-domain measure. Intelligence, 43, 52-64. doi:10.1016/j.intell.2014.01.004

Damasio, A. R., Everitt, B. J., \& Bishop, D. (1996). The somatic marker hypothesis and the possible functions of the prefrontal cortex [and discussion]. Philosophical Transactions of the Royal Society B: Biological Sciences, 351(1346), 1413-1420. doi:10.1098/rstb.1996.0125.

Demaree, H. A., Burns, K. J., \& DeDonno, M. A. (2010). Intelligence, but not emotional intelligence, predicts Iowa Gambling Task performance. Intelligence, 38(2), 249-254. doi:10.1016/ j.intell.2009.12.004

Epstein, S., Pacini, R., Denes-Raj, V., \& Heier, H. (1996). Individual differences in intuitive-experiential and analytical-rational thinking styles. Joumal of Personality and Social Psychology, 71(2), 390. doi:10.1037/0022-3514.71.2.390

Grasman, R., \& Wagenmakers, E. (2005). A DHTML implementation of the Iowa Gambling Task. Retrieved from http://purl.oclc.org/NET/rgrasman/jscript/IowaGamblingTask

Hastie, R., \& Dawes, R. M. (2010). Rational choice in an uncertain world: The psychology of judgment and decision making. USA: Sage.

Kahneman, D. (2013). Dumai medlenno... reshai bystro [Thinking, fast and slow]. Moscow: AST. (Transl. of: Kahneman, D.(2011). Thinking, fast and slow. New York: Farrar, Straus and Giroux).

Kornilov, S. A., \& Grigorenko, E. L. (2010). Metodicheskij kompleks dlya diagnostiki analiticheskih, tvorcheskih i prakticheskih sposobnostej [The assessment battery for analytical, creative, and practical abilities]. Psihologicheskij Zhurnal, 31(2), 90-103.

Kornilov, S. A., Krasnov, E., Kornilova, T. V., \& Chumakova, M. A. (2015). Individual differences in performance on Iowa Gambling Task are predicted by tolerance and intolerance for uncertainty. In G. Airenti, B. G. Bara, \& G. Sandini (Eds.), Proceedings of the EuroAsianPacific Joint Conference on Cognitive Science (Vol. 1419, pp. 728-731). Torino, Italy. Retrieved from http://ceurws.org/Vol-1419/paper0121.pdf

Kornilova, T. V. (2016a). Intellektualno-lichnostmyj potencial cheloveka v usloviyah neopredelennosti $i$ riska [Intellectual and personality potential of a person under the conditions of uncertainty and risk ]. Saint Petersburg: Nestor-Istoriya.

Kornilova, T. V. (2016b). Psihologija vybora kak myslitel'noe i lichnostnoe oposredstvovanie preodolenija neopredelennosti [Psychology of choice and decision making as cognitive and personality moderated overcoming of uncertainty], Psihologicheskij Zhurnal, 37(3), 113-124.

Kornilova, T. V., \& Razvaliaeva, A. U. (2017). Aprobatsija russkojazychnogo variant polnogo oprosnika S. Epstaina "Racionalnyi-Opytnyi" (Rational-Experiental Inventory) [The rationality and intuition scales in S. Epstein's questionnaire REI (Russian approbation of the full version)]. Psikhologicheskii Zhumal, 38(3), 92-107. doi:10.7868/S0205959217030084.

Kornilova, T. V., \& Razvalyaeva, A. U. (2016). Psychological mindedness is related to cognitive strategies of choice and decision making in a prognostic task. In Yu. I. Alexandrov, K. V. Anokhin (Eds.), Sed'maya mezhdunarodnaya konferentsiya po kognitionoy nauke: Tezisy dokladov [Seventh International Conference on Cognitive Science: Proceedings] (pp. 63-65). Moscow: Institute of Psychology of RAS. 
Krasavtseva, Y., \& Kornilova, T. (2016). Svojstva Temnoj triady v reguljacii strategij prinjatija reshenij (na materiale igrovoj zadachi Ajova - IGT) [The Dark Triad Traits in the regulation of decisionmaking strategies]. Vestnik Moskovskogo Gosudarstvennogo Oblastnogo Universiteta. Serija: Psihologicheskie Nauki, [Bulletin of Moscow Regional State University. Series: Psychology], 2, 22-33. doi:10.18384/2310-7235-2016-2-22-33

Medvedeva, T. I., Enikolopov, S.N., \& Enikolopova, E.V. (2013). Gipoteza somaticheskih markerov Damasio i igrovaja zadacha (IGT): obzor [Damasio's Somatic Markers Hypothesis and Iowa Gambling Task (review)]. Psihologicheskie Issledovaniya, 6(32), 10. Retrieved from http://psystudy.ru/index.php/num/2013v6n32/912-medvedeva32.html

Paulhus, D. L., \& Williams, K. M. (2002). The dark triad of personality: narcissism, Machiavellianism, and psychopathy. Journal of Research in Personality, 36(6), 556-563. doi:10.1016/S00926566(02)00505-6

Smirnov, S. D., Chumakova, M. A., \& Kornilova, T. V. (2016). Obraz mira v dinamicheskom kontrole neopredelennosti [The world image in dynamic control of uncertainty]. Voprosy Psikhologii, 4, $3-13$.

Stepanosova, O. V., \& Kornilova, T. V. (2006). Motivacija i intuicija v reguljacii verbal'nyh prognozov pri prinjatii reshenij [Motivation and intuition in verbal predictions regulation in decision making]. Psikhologicheskii Zhumal, 27(2), 60-68.

Webb, C. A., DelDonno, S., \& Killgore, W. D. S. (2014). The role of cognitive versus emotional intelligence in Iowa Gambling Task performance: What's emotion got to do with it? Intelligence, 44,

Tatiana V. Kornilova - professor, Department of Psychology, Lomonosov Moscow State University, D.Sc.

Research area: psychology of thinking and decision-making, personality psychology,

psychology of risk, self regulation, intellectual-personality potential.

E-mail: tvkornilova@mail.ru

Maria A. Chumakova - associate professor, School of psychology, Department of Social sciences, National Research University Higher School of Economics, Ph.D.

Research area: decision making, intelligence and personality potential, individual differences in intelligence and attitude towards uncertainty.

E-mail: mchumakova@hse.ru

Sergey A. Kornilov - research fellow, Department of Psychology, Saint-Petersburg State University, Ph.D.

Research area: psychometrics, intelligence, developmental disorders.

E-mail: sa.kornilov@gmail.com 